\title{
Truncated disc surface brightness profiles produced by flares
}

\author{
Alejandro Borlaff ${ }^{1,3}$, M. Carmen Eliche-Moral ${ }^{2}$, John Beckman ${ }^{1,3,4}$ \\ and Joan Font ${ }^{1,3}$ \\ ${ }^{1}$ Instituto de Astrofísica de Canarias, La Laguna, Tenerife, Spain, email: asborlaff@iac.es \\ ${ }^{2}$ Departamento de Astrofísica y CC. de la Atmósfera, Univ. Complutense de Madrid, Spain \\ ${ }^{3}$ Facultad de Física, Universidad de La Laguna, Spain \\ ${ }^{4}$ Consejo Superior de Investigaciones Científicas, Spain
}

\begin{abstract}
Previous studies have discarded that flares in galactic discs may explain the truncation that are frequently observed in highly-inclined galaxies (Kregel et al. 2002). However, no study has systematically analysed this hypothesis using realistic models for the disc, the flare and the bulge. We derive edge-on and face-on surface brightness profiles for a series of realistic galaxy models with flared discs that sample a wide range of structural and photometric parameters across the Hubble Sequence, accordingly to observations. The surface brightness profile for each galaxy model has been simulated for edge-on and face-on views to find out whether the flared disc produces a significant truncation in the disc in the edge-on view compared to the face-on view or not. In order to simulate realistic images of disc galaxies, we have considered the observational distribution of the photometric parameters as a function of the morphological type for three mass bins $\left(10<\log _{10}\left(M / M_{\odot}\right)<10.7,10.7<\log _{10}\left(M / M_{\odot}\right)<11\right.$ and $\left.\log _{10}\left(M / M_{\odot}\right)>11\right)$, and four morphological type bins (S0-Sa, Sb-Sbc, Sc-Scd and Sd-Sdm). For each mass bin, we have restricted the photometric and structural parameters of each modelled galaxy to their characteristic observational ranges $\left(\mu_{0, \text { disc }}, \mu_{\text {eff }, \text { bulge }}, B / T, M_{\text {abs }}, r_{\text {eff }}, n_{\text {bulge }}, h_{\mathrm{R}, \text { disc }}\right)$ and the flare in the $\operatorname{disc}\left(h_{\mathrm{z}, \mathrm{disc}} / h_{\mathrm{R}, \mathrm{disc}}, \partial h_{\mathrm{z}, \mathrm{disc}} / \partial R\right.$, see de Grijs \& Peletier 1997, Graham 2001, López-Corredoira et al. 2002, Yoachim \& Dalcanton 2006, Bizyaev et al. 2014, Mosenkov et al. 2015).

Contrary to previous claims, the simulations show that realistic flared disks can be responsible for the truncations observed in many edge-on systems, preserving the profile of the nonflared analogous model in face-on view. These breaks reproduce the properties of the weak-tointermediate breaks observed in many real Type-II galaxies in the diagram relating the radial location of the break $\left(R_{\mathrm{brkII}}\right)$ in units of the inner disk scale-length with the break strength $S$ (Laine et al. 2014). Radial variation of the scale-height of the disc (flaring) can explain the existence of many breaks in edge-on galaxies, especially of those with low break strengths $S=\log _{10} \frac{h_{o}}{h_{i}} \sim[-0.3,-0.1]$.
\end{abstract}

Keywords. Galaxies: formation - galaxies: fundamental parameters - galaxies: structure

\section{References}

Bizyaev, D. V., Kautsch, S. J., Mosenkov, A. V., Reshetnikov, V. P., Sotnikova, N. Y., Yablokova, N. V., \& Hillyer, R. W. 2014, ApJ, 787, 24

de Grijs, R. \& Peletier, R. F. 1997, A\& A, 320, L21

Graham, A. W. 2001, AJ, 121, 820

Kregel, M., van der Kruit, P. C., \& de Grijs, R. 2002, MNRAS, 334, 646

Laine, J., et al. 2014, MNRAS, 441, 1992

López-Corredoira, M., Cabrera-Lavers, A., Garzón, F., \& Hammersley, P. L. 2002, A\& A, 394, 883

Mosenkov, A. V., Sotnikova, N. Y., Reshetnikov, V. P., Bizyaev, D. V., \& Kautsch, S. J. 2015, MNRAS, 451, 2376

Yoachim, P. \& Dalcanton, J. J. 2006, AJ, 131, 226 